\title{
Stochastic non-minimal state space control with application to automated drug delivery
}

\author{
Emma D. Wilson*, Quentin Clairon ${ }^{\dagger}$ and C. James Taylor* \\ * Engineering Department, Lancaster University, Lancaster, UK, LAl 4YW \\ Email:e.d.wilson1@lancaster.ac.uk, c.taylor@lancaster.ac.uk \\ ${ }^{\dagger}$ School of Mathematics, Statistics and Physics, Newcastle University, Newcastle upon Tyne, UK, NE1 7RU \\ Email: quentin.clairon@newcastle.ac.uk
}

\begin{abstract}
This paper shows how a standard proportionalintegral-plus controller, based on a non-minimal state space (NMSS) design, can be extended to reduce the effects of measurement noise and so yield smoother control inputs for automated drug delivery control applications. Use of a NMSS model for state variable feedback control design, in which all the states are based on the directly measured input and output variables, removes the need for state estimation. Nonetheless, a stochastic NMSS form, with a Kalman filter, can optionally be introduced to reduce the effect of measurement noise and so yield smoother control inputs. In this case, the Kalman filter attenuates measurement noise but does not address state disturbances. In this article, we propose a modification to the stochastic NMSS control system to enable the elimination of such state disturbances. This involves further extending the non-minimal state vector to include additional terms based on the innovations. We compare the deterministic, stochastic and extended stochastic NMSS controllers via a simulation of the control of anaesthesia using propofol.
\end{abstract}

Keywords-Adaptive Treatment; Stochastic control; Kalman Filter; Non-Minimum State Space (NMSS); ProportionalIntegral-Plus (PIP); Anaesthesia

\section{INTRODUCTION}

We apply non-minimal state space (NMSS), proportionalintegral-plus (PIP) control to a simulated drug delivery example, namely the control of anaesthesia using propofol. During anaesthetic drug delivery, the patient is continually monitored and the combination of drugs adjusted to ensure normal vital signs are maintained during loss of consciousness [1]. The advantages of using automatic controllers in anaesthesia are recognised [2]. However, it remains a challenging control problem, due to inter-patient variability, poor understanding of anaesthetic drug mechanisms and difficulties in sensing. Nonetheless, control theory has been previously used to develop (mainly proportional-integral (PI) and proportional integral derivative (PID)) algorithms to control anaesthesia, with examples including references: [1], [2], [3], [4].

PIP control provides a flexible and straightforward, model-based approach for control system design [5], [6], [7], and can be interpreted as a logical extension of conventional PI or PID control. Such PIP controllers have been utilised in a range of application areas e.g. [8], [9], [10],
[11] and they also show promise for biomedical control applications [12]. In the discrete-time case, the non-minimal state vector consists of the present and past sampled values of the output, past sampled values of the input and, commonly, an integral-of-error term. The length of the state vector is not constrained, hence NMSS models can be used to provide more design freedom than minimal forms [8], [6], [7]. The use of NMSS models also removes the need for state estimation; see e.g. [13] and the citations above.

State estimation typically forms an integral part of conventional, minimal state space control design [14]. Here, the system behaviour is described by a set of linearly independent state variables [15]. In the minimal case, the state vector is as small as possible and the states are not necessarily available for direct measurement. Hence, state estimation is used to access state information for feedback control. Common methods include, for example, the ubiquitous Kalman Filter (KF) and various extensions [16], [17], [18], particle filters [19], [20], sliding mode observers [21], [22] and neural networks [23].

Although there is no requirement for a state reconstructor with NMSS control, state estimation can still be introduced to remove measurement noise from the optimal estimate of the output, and so reduce the effect of this noise on the input. A smoother input signal can be advantageous for automated drug delivery, as it results in smaller dose changes at each instant. Consequently, the KF can be expressed in NMSS form and, exploiting the separation theorem in the usual manner [24], a PIP control law applied to the estimated state.

Previous research into such stochastic NMSS-PIP control has focused on Auto-Regressive, Moving-Average eXogenous variable (ARMAX) models [8], [6], [7]. Here, by contrast, we consider control of a system that is described by a full Box-Jenkins (BJ) model. Whilst a recent article by the present authors considered NMSS representations of the BJ model from a system identification viewpoint [25], the present article focuses on the design and performance of the controller in a simulated biomedical control example.

State estimation addresses measurement noise, with the state disturbances effectively 'passed' by the KF. Hence, a second contribution of this article is to extend the non- 
minimal state vector to include estimates of such disturbances. Although these states are uncontrollable in system design terms, they enable the effect of state disturbances to be removed from the closed-loop response. Deterministic PIP control, stochastic PIP control with state estimation, and the new approach based on an extended state vector, are all described in section II. We subsequently apply these controllers to the specific problem of the control of anasthesia using propofol in section III.

\section{Non Minimal State Space Control}

We consider the control of a generalised BJ system model, in which the sampled output $y(k)$ is expressed in terms of the sampled input $u(k)$ and Gaussian noise $\eta(k)$ in discretetime Transfer Function (TF) form, as follows,

$$
y(k)=\frac{B\left(z^{-1}\right)}{A\left(z^{-1}\right)} u(k)+\frac{D\left(z^{-1}\right)}{C\left(z^{-1}\right)} \eta(k)
$$

where $z^{-1}$ is the backward shift operator, i.e. $z^{-1} u(k)=$ $u(k-1), A\left(z^{-1}\right)=1+a_{1} z^{-1}+a_{2} z^{-2}+\cdots+a_{n} z^{-n}$, $B\left(z^{-1}\right)=b_{1} z^{-1}+b_{2} z^{-2}+\cdots+b_{m} z^{-m}, C\left(z^{-1}\right)=1+$ $c_{1} z^{-1}+c_{2} z^{-2}+\cdots+c_{p} z^{-p}$ and $D\left(z^{-1}\right)=1+d_{1} z^{-1}+$ $d_{2} z^{-2}+\cdots+d_{q} z^{-q}$. Here, $a_{i}(i=1 \ldots n), b_{i}(i=1 \ldots m)$, $c_{i}(i=1 \cdots p)$ and $d_{i}(i=1 \cdots q)$ are constant coefficients.

The full polynomial BJ model, Eq. (1), can be expressed in stochastic state space form using an extended nonminimal state vector. The KF representation of this NMSS system, assuming convergence of the Kalman gain vector $\boldsymbol{L}$, was recently considered by [25], building on earlier results by e.g. [26]. Hence, the KF can be implemented in various polynomial forms. In the following, we consider three main control structures: A) standard deterministic PIP control, B) PIP control with state estimation and C) novel PIP control with state estimation and innovations in the state vector.

\section{A. Deterministic PIP control}

The standard NMSS state vector [5] is given by $\boldsymbol{x}(k)=$ $[y(k) \cdots y(k-n+1) u(k-1) \cdots u(k-m+1) z(k)]^{\prime}$

where' represents the transpose and, in recursive terms, the integral-of-error state is $z(k)=z(k-1)+\left(y_{d}(k)-y(k)\right)$ in which $y_{d}(k)$ is the command input. For deterministic PIP control, the state variable feedback control law takes the usual form $u(k)=-\boldsymbol{k} \boldsymbol{x}(k)$, where $\boldsymbol{k}$ is a $n+m$ control gain vector,

$$
\boldsymbol{k}=\left[\begin{array}{llllllll}
f_{0} & f_{1} & \cdots & f_{n-1} & g_{1} & \cdots & g_{m-1} & -k_{I}
\end{array}\right]
$$

These gains are selected by the designer to achieve desired closed-loop characteristics, for example, by using optimal linear quadratic (LQ) control or pole placement [7]. In this article we design a feedback gain vector that minimises the following LQ cost function using standard methods,

$$
J=\sum_{k=1}^{\infty} \boldsymbol{x}(k) \boldsymbol{Q} \boldsymbol{x}(k)+q_{u}\left(u(k)^{2}\right)
$$

Exploiting the special structure of the NMSS model, common weighting values are used for the output, input and integral-of-error states, as follows,

$$
\boldsymbol{Q}=\operatorname{diag}\left(\begin{array}{lllllll}
q_{y} & \cdots & q_{y} & q_{u} & \cdots & q_{u} & q_{e}
\end{array}\right)
$$

with,

$$
q_{y}=\frac{w_{y}}{n}, \quad q_{u}=\frac{w_{u}}{m}, \quad q_{e}=w_{e}
$$

where $w_{y}, w_{u}$ and $w_{e}$ are three scalar weights, selected by the designer [7]. Figure 1a illustrates the resulting PIP control system in block diagram form, where,

$$
\begin{array}{r}
G\left(z^{-1}\right)=1+g_{1}+\cdots+g_{m-1} z^{-m+1} \\
F\left(z^{-1}\right)=f_{0}+f_{1} z^{-1}+\cdots+f_{n-1} z^{-n+1}
\end{array}
$$

\section{B. PIP control with state estimation}

Linear Quadratic Gaussian (LQG) control utilises the estimated state $\hat{\boldsymbol{x}}(k)$ in place of $\boldsymbol{x}(k)$ i.e. $u(k)=-\boldsymbol{k} \hat{\boldsymbol{x}}(k)$. Using the separation principal [24], the stochastic control law is simply the vector of control gains from section II-A applied to the optimal estimate of the states. For the BJ model (1), the optimal estimate of the output is determined from the measured input and output signals as follows [25],

$$
\hat{y}(k)=\frac{\tilde{B}\left(z^{-1}\right) \tilde{C}\left(z^{-1}\right)}{\tilde{A}\left(z^{-1}\right) \tilde{D}\left(z^{-1}\right)} u(k)+\frac{\tilde{C}\left(z^{-1}\right)-\tilde{D}\left(z^{-1}\right)}{\tilde{D}\left(z^{-1}\right)} y(k)
$$

where $\tilde{A}\left(z^{-1}\right), \tilde{B}\left(z^{-1}\right), \tilde{C}\left(z^{-1}\right), \tilde{D}\left(z^{-1}\right)$ represent estimates of the system polynomials. The state vector $\tilde{\boldsymbol{x}}(k)$ is expressed in terms of the estimated output $\hat{y}(k)$, known input $u(k)$ and their past values. The stochastic PIP controller, with the KF implemented in this polynomial form, is shown in Fig. 1b.

A major drawback of this control strategy is that the stochastic model (1) does not account for non-stationary (time-varying) disturbance inputs to the system. Therefore, if the control structure in Fig. 1b is used on a system subject to load disturbances (which arise when the estimated model is incorrect), steady state errors occur. This issue can be rectified using one of the following methods:

1) Use of the measured output: A somewhat ad hoc but straightforward and practically useful approach is to utilise the measured output in place of the estimate in the definition of the integral-of-error state $z(k)$ (similar to the deterministic PIP controller). This yields the control system illustrated by Fig. 1c. The steady state gain of the stochastic component is zero, as required for elimination of steady state errors.

2) Random walk state: To address the problem of nonstationary disturbances, a random walk state can be introduced into the NMSS form. The corresponding control system is given by Fig. 1d. The associated state equations 


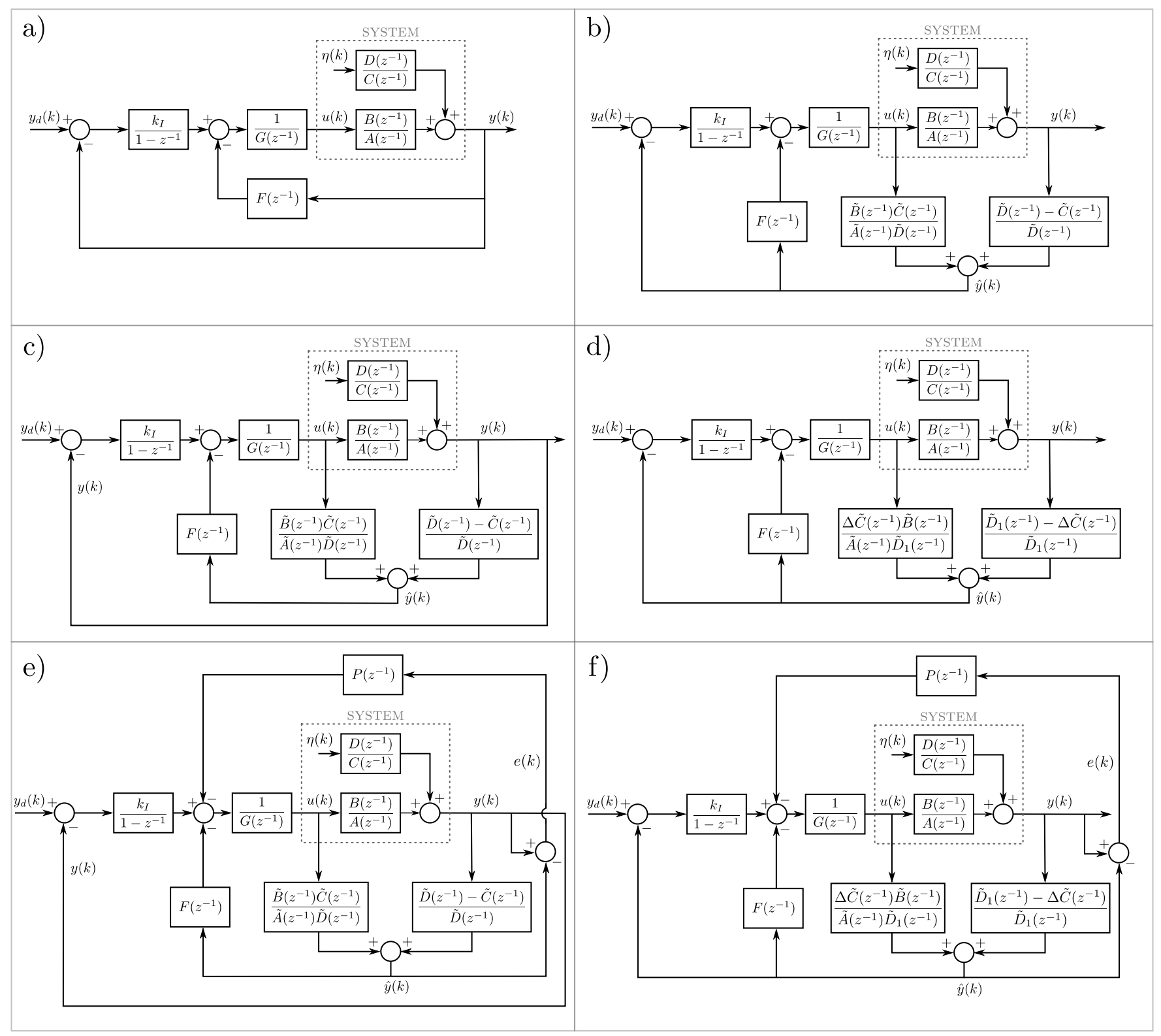

Figure 1. PIP control of a plant with stochastic disturbances: a) deterministic control; b) state estimation and integral action operating on the estimated output; c) state estimation and integral action operating on the measured output; d) state estimation and a random walk state to ensure integral action, in which $\Delta=1-z^{-1}$ and $\tilde{D}_{1}\left(z^{-1}\right)=\tilde{D}\left(z^{-1}\right) \Delta+l_{n+1} \tilde{C}\left(z^{-1}\right)$; e) state estimation and use of the innovations in the state vector, with the integral action operating on the measured output; f) state estimation and use of the innovations in the state vector, and a random walk state to ensure integral action.

are,

$$
\begin{array}{r}
{\left[\begin{array}{c}
\hat{\boldsymbol{x}}(\boldsymbol{k}) \\
r(k)
\end{array}\right]=\left[\begin{array}{cc}
\boldsymbol{F} & \mathbf{0} \\
\mathbf{0}^{\prime} & 1
\end{array}\right]\left[\begin{array}{c}
\hat{\boldsymbol{x}}(\boldsymbol{k}-\mathbf{1}) \\
r(k-1)
\end{array}\right]+\left[\begin{array}{c}
\boldsymbol{g} \\
0
\end{array}\right] u(k)+\left[\begin{array}{c}
\boldsymbol{L} \\
l_{n+1}
\end{array}\right] \eta(k)} \\
y(k)=\left[\begin{array}{ll}
\boldsymbol{h} & 1
\end{array}\right]\left[\begin{array}{c}
\hat{\boldsymbol{x}}(\boldsymbol{k}) \\
r(k)
\end{array}\right]+\eta(k)
\end{array}
$$

where $\boldsymbol{F}$ and $\boldsymbol{h}$ are the NMSS transition matrix and observation vector respectively [7] (the full state space model is omitted for brevity), $\mathbf{0}$ is a column vector of zeros with length equal to the length of $\boldsymbol{F}$ and $l_{n+1}$ is the gain associated with the new state $r(k)$. Hence, the random walk state is expressed in terms of the disturbance as follows $r(k)=\left(1-z^{-1}\right)^{-1} l_{n+1} \eta(k)$ and the optimal estimate of the output becomes,

$\hat{y}(k)=\frac{\tilde{D}_{1}\left(z^{-1}\right)-\Delta \tilde{C}\left(z^{-1}\right)}{\tilde{D}_{1}\left(z^{-1}\right)} y(k)+\frac{\Delta \tilde{C}\left(z^{-1}\right) \tilde{B}\left(z^{-1}\right)}{\tilde{A}\left(z^{-1}\right) \tilde{D}_{1}\left(z^{-1}\right)} u(k)$

where $\Delta=1-z^{-1}$ and $\tilde{D}_{1}\left(z^{-1}\right)=\tilde{D}\left(z^{-1}\right) \Delta+$ $l_{n+1} \tilde{C}\left(z^{-1}\right)$. 


\section{PIP control with state estimation and extended state}

A new way to introduce stochastic disturbances into the NMSS formulation is proposed in this article. Here, the state vector is extended to explicitly include the $\mathrm{KF}$ innovations sequence, $e(k)=y(k)-\hat{y}(k)$, as follows,

$$
\begin{aligned}
& \boldsymbol{x}(k)=[y(k) \cdots y(k-n+1) u(k-1)
\end{aligned}
$$

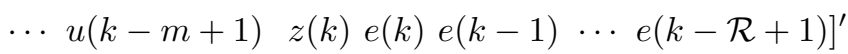

where, for the $\mathrm{BJ}$ model $(1), \mathcal{R}=n+\max (p, q)$ is the order of $A\left(z^{-1}\right)\left(D\left(z^{-1}\right)-C\left(z^{-1}\right)\right)$. In polynomial form, the new control element is $R\left(z^{-1}\right)=r_{0}+r_{1} z^{-1}+\cdots+r_{\mathcal{R}-1} z^{-\mathcal{R}+1}$ and the associated control structures are illustrated by Fig. 1e or Fig. 1f, where $P\left(z^{-1}\right)$ depends on the chosen method for determining $R\left(z^{-1}\right)$, as described below.

1) LQ optimisation: Although the innovation states are not controllable from the input, they are introduced into the NMSS model to provide extra information for state variable feedback. In the case of LQ design, $P\left(z^{-1}\right)=$ $R\left(z^{-1}\right)=r_{0}+\cdots+r_{\mathcal{R}-1} z^{-\mathcal{R}+1}$ (in Fig. 1e-f) is used to exploit information on the estimated disturbance, in order to predict and correct for this disturbance. In a similar manner to deterministic feed-forward control [8] and command anticipation [6], the NMSS model is uncontrollable by standard definitions. Hence, the choice of cost function weights associated with the innovation states have no effect on the control gains and are set to zero.

2) Exact cancellation: Alternatively, the extra control gains are chosen to explicitly eliminate stochastic disturbances by cancellation. If the system model is assumed correct, then the innovations $e(k)$ have the same statistical properties as the disturbance $\eta(k)$. The corresponding input and output variables, expressed in Transfer Function form as functions of the command input $y_{d}(k)$ and noise $\eta(k)$, are,

$$
\begin{array}{r}
u(k)=\frac{A k_{I}}{A G \Delta+B\left(\Delta F+k_{I}\right)} y_{d}(k) \\
+\frac{A(C-D)\left(\Delta F+k_{I}\right)-C A \Delta P}{C\left(A G \Delta+B\left(\Delta F+k_{I}\right)\right)} \eta(k) \\
y(k)=\frac{B k_{I}}{A G \Delta+B\left(\Delta F+k_{I}\right)} y_{d}(k) \\
+\frac{D A G \Delta+C B\left(\Delta F+k_{I}\right)-C B \Delta P}{C\left(A G \Delta+B\left(\Delta F+k_{I}\right)\right)} \eta(k)
\end{array}
$$

where the operator notation $z^{-1}$ has been omitted for clarity. From Eq. (9), the input has zero stochastic component when $C\left(z^{-1}\right) \Delta P\left(z^{-1}\right)=\left(C\left(z^{-1}\right)-D\left(z^{-1}\right)\right)\left(\Delta F\left(z^{-1}\right)+k_{I}\right)$. Therefore, if in Fig. 1e-f, $P\left(z^{-1}\right)=R\left(z^{-1}\right) /\left(C\left(z^{-1}\right) \Delta\right)$ where $R\left(z^{-1}\right)=\left(\tilde{C}\left(z^{-1}\right)-\tilde{D}\left(z^{-1}\right)\right)\left(\Delta F\left(z^{-1}\right)+k_{I}\right)$ to determine the gains, the input will have no stochastic component.
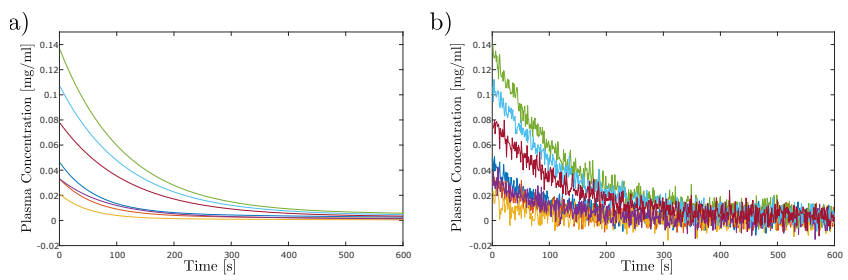

Figure 2. Impulse response of each model given in Table I, showing a) noise free case and b) with additive white noise $\eta$.

\section{Control of Clinical Anasthesia}

A basic PK-PD pharmacological model can be used to model the relationship between the administered aesthetic dose and the effect of the patient in terms of hypnosis and analgesia [2]. The PK-PD model consists of a pharmacokinetic $(\mathrm{PK})$ model that is used to describe the evolution of the drug plasma concentration following the administration of a drug, and a pharmacodynamic (PD) model that is used to express the observed effect of a drug as a function of the plasma concentration. In this contribution, we just consider the PK part of the model and hence the control of the drug plasma concentration via administered doses in the presence of measurement noise. A three compartment model, expressed as a third order TF is used [2]. The parameters of the discrete-time propofol TF model, based on equation (1) with $n=m=3$, are presented in Table I (various options for the noise model component are discussed later). These parameters were converted from the continuous-time equivalents given by [2] using the Matlab function $c 2 \mathrm{~d}$. The impulse response of each model with and without white noise, for example, is shown in Fig. 2.

\section{A. White Noise Inputs}

The controlled response in the presence of white noise inputs is first considered i.e. based on equation (1) with $C\left(z^{-1}\right)=D\left(z^{-1}\right)$. The nominal plant was simulated using the model from study II) in Table I, with plant model inaccuracies represented by designing controllers based on models from each of the other studies (I, III, IV, V, VI, VII). In these simulations, the command input $y_{d}(k)$ is a step change from zero to 0.1 , whilst the noise $\eta(k)$ is a zero mean, Gaussian signal with variance 0.005 . LQ controllers are designed as described in section II-A, with weights of $w_{y}=w_{u}=1, w_{e}=0.01$ in all cases. For the relevant control structures, the gain of the random walk $l_{n+1}=0.1$.

The results for each control strategy (i.e. the strategies shown by Fig. 1) are illustrated in Fig. 3. When the model is accurate, state estimation (SE) is successfully used to reduce the variance of the input signal, as shown by comparing Fig. 3a and 3b. However, the effect of an incorrect system model is demonstrated in Fig 3c, which highlights steady state errors for all but the nominal (ideal model) response. As discussed in section II-B, integral action is lost when 


\begin{tabular}{|c|c|c|c|c|c|c|c|}
\hline Label & Study & & $\begin{array}{lll}b_{1} & b_{2} & b_{3}\end{array}$ & & \multicolumn{3}{|c|}{$\left[\begin{array}{lll}a_{1} & a_{2} & a_{3}\end{array}\right]$} \\
\hline I) & Schnider et al. [27] 30yrs, $50 \mathrm{~kg}, 1.7 \mathrm{~m}$ & 1.9094 & -3.4713 & $1.5631]$ & -2.2994 & 1.6505 & -0.3510 \\
\hline II) & Schnider et al. [27] 30yrs, $70 \mathrm{~kg}, 1.7 \mathrm{~m}$ & 1.3427 & -2.4410 & 1.0992 & -2.2826 & 1.6213 & -0.3386 \\
\hline III) & Schnider et al. [27] $30 \mathrm{yrs}, 110 \mathrm{~kg}, 1.7 \mathrm{~m}$ & 0.7744 & -1.4079 & $0.6340]$ & -2.1903 & 1.4584 & -0.2679 \\
\hline IV) & Schnider et al. [27] 70yrs, $70 \mathrm{~kg}, 1.7 \mathrm{~m}$ & 1.4693 & -2.6705 & $1.2020]$ & {$[-2.3621$} & 1.7850 & -0.4228 \\
\hline V) & Schüttler et al. [28] 30yrs, $50 \mathrm{~kg}$ & {$[6.4508$} & -12.5810 & 6.1310 & -2.5597 & 2.1304 & -0.5707 \\
\hline VI) & Schüttler et al. [28] 30yrs, $70 \mathrm{~kg}$ & 5.0902 & -9.9244 & $4.8350]$ & -2.5658 & 2.1429 & -0.5770 \\
\hline VII) & Schüttler et al. [28] 30yrs, $110 \mathrm{~kg}$ & {$[3.7180$} & -7.2455 & $3.5282]$ & {$[-2.5717$} & 2.1550 & -0.5833 \\
\hline
\end{tabular}

Table I

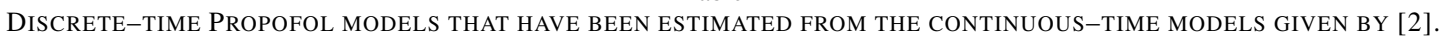

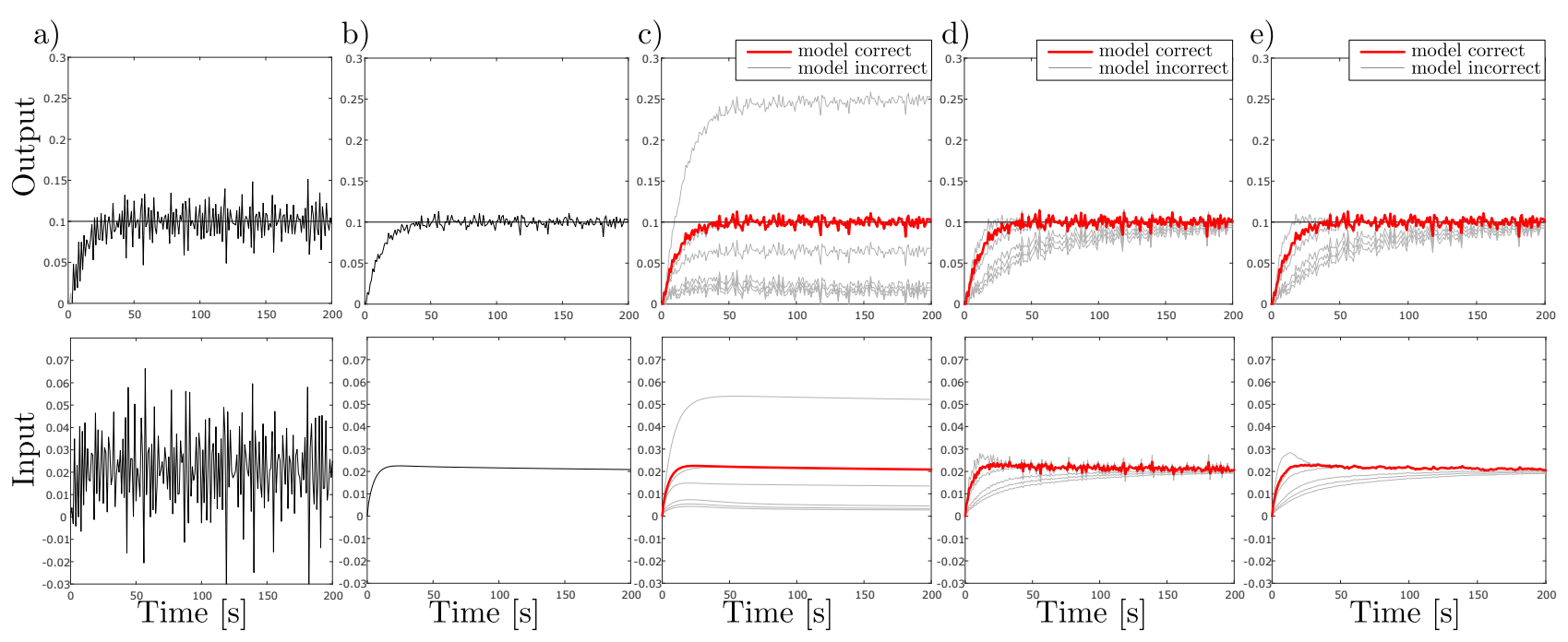

Figure 3. Output (top) and control input (bottom) with white measurement noise: a) No state estimation (SE) (Fig 1a), model correct; b) Just SE (Fig 1b), model correct; c) Just SE (Fig 1b), model incorrect; d) SE and random walk state (Fig 1d), model incorrect; e) SE and feedback $y$ (Fig 1c), model incorrect.

\begin{tabular}{ccccc} 
Control & \multicolumn{2}{c}{ Correct model } & \multicolumn{2}{c}{ Incorrect model } \\
structure & MSE & uvar & MSE & uvar \\
\hline & $1 e^{-3} \times$ & $1 e^{-3} \times$ & $1 e^{-3} \times$ & $1 e^{-3} \times$ \\
Fig. 1a & 0.194 & 0.177 & 0.172 & 0.083 \\
Fig. 1b & 0.028 & 0.005 & 3.458 & 0.004 \\
Fig. 1c & 0.067 & 0.039 & 0.056 & 0.029 \\
Fig. 1d & 0.080 & 0.050 & 0.074 & 0.046 \\
Fig. 1f with LQ optimisation & 0.030 & 0.009 & 0.032 & 0.007 \\
Fig. 1f with exact cancellation & 0.030 & 0.001 & 0.034 & 0.001 \\
& Table II & & &
\end{tabular}

MEAN SQUARE ERROR (MSE) AND INPUT VARIANCE (UVAR) FOR DIFFERENT CONTROL STRATEGIES WITH COLOURED NOISE INPUTS.

including basic state estimation, hence the very poor results associated with this control structure (Fig. 1b). These steady state tracking errors are successfully eliminated by including a random walk state (Fig. 1d) or by feeding back the actual output (Fig. 1c), as demonstrated by the simulation results in Fig. 3d,e.

\section{B. Coloured Noise Inputs}

The controlled response in the presence of coloured noise inputs is next considered. Noise polynomials $C\left(z^{-1}\right)=$ $1-0.4 z^{-1}$ and $D\left(z^{-1}\right)=1+0.1 z^{-1}$ are chosen for illustrative purposes. Selected simulation results are illustrated in Fig. 4, with the performance metrics summarised in Table II. Performance was evaluated as the variance of the control input $u(k)$ and the mean squared error (MSE) of $y_{d}(k)-y(k)$ at steady state (values taken between 10 to 20 minutes into the simulation). Experiments that yield an unstable response (because of modelling errors) are omitted from the metrics.

For non-white noise inputs, even if the system model is accurate, state estimation alone does not result in smooth input signals; see Fig. 4b. The KF is designed to attenuate 'measurement' noise (estimated as $e(k)$ ), however, it passes state disturbances $(\boldsymbol{L} e(k-1)$ in the innovations representation). Therefore, if the noise is not white, i.e. $D\left(z^{-1}\right) \neq C\left(z^{-1}\right)$, the estimated output still contains coloured noise $\left(D\left(z^{-1}\right)-C\left(z^{-1}\right)\right) / C\left(z^{-1}\right)$. The noise on the output naturally yields an associated noisy input signal. This applies even in the ideal case when the model 
a)
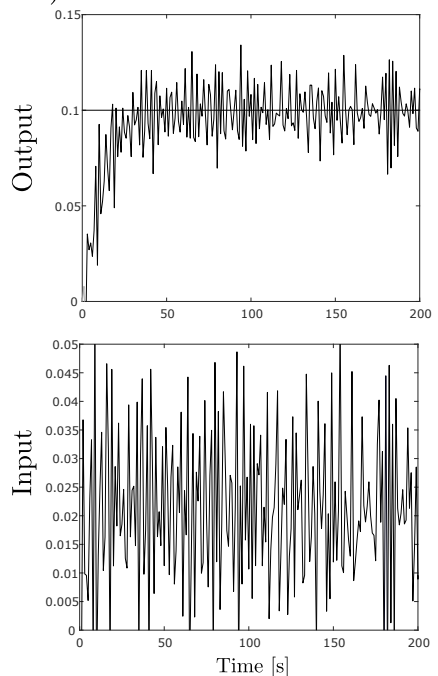

b)
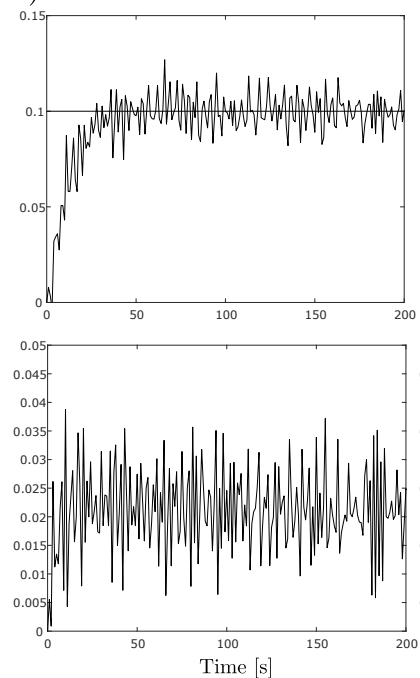

c)

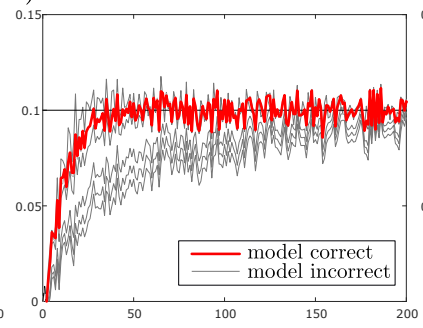

d)

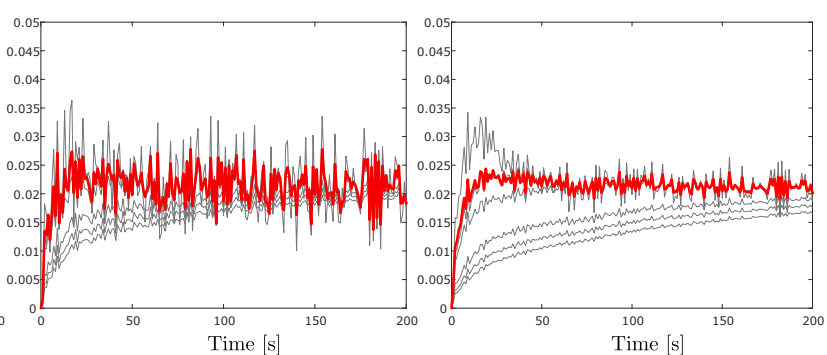

Figure 4. Output (top) and control input (bottom) with coloured measurement noise: a) No state estimation (SE) (Fig 1a), model correct; b) Just SE (Fig 1b), model correct; c) SE with random walk and innovations in state vector (Fig 1f), using LQ optimisation for the extra terms; d) SE with random walk and innovations in state vector (Fig 1f), using exact cancellation for the extra terms.

coefficients are exactly known. In the context of system identification, such issues are discussed in more detail by Young [29].

To address this problem, the proposal in section II-C is to extend the state vector with the estimated innovations. For the present model, $\mathcal{R}=4$, hence these states are $[e(k)$, $e(k-1), e(k-2), e(k-3)]$. Gains corresponding to the extra terms are estimated using both LQ optimisation and the exact cancellation approach. For an exactly known model, the latter approach reduces the variance of the input to almost zero as would be expected: see Fig. 4d. LQ optimisation can also be used to reduce the input variance, as shown by Fig. 4c; however, for certain models these reductions are not very significant. A generalised matrix for $\boldsymbol{Q}$ in equation (3) could help to address this problem and is the subject of ongoing research by the authors.

\section{CONCLusions}

A state observer is not required for state variable feedback when using NMSS models but can optionally be introduced to help smooth the input signals. The use of smoothly varying input signals is important in many biological control applications. In stochastic NMSS control, a KF addresses the effects of measurement noise. However, if the system is described using a noise model $D\left(z^{-1}\right) / C\left(z^{-1}\right)$ and $D\left(z^{-1}\right) \neq C\left(z^{-1}\right)$ then there is a filtered component to the noise, which appears as state noise and cannot be removed using the KF alone. Using the NMSS modelling and control framework, we have proposed extending the state vector to include estimates of the disturbance signal and have evaluated the new approach via simulation for anasthesia control using propofol. The approach is applicable to other biomedical and conventional engineering control applications. The method is shown to be potentially very effective at reducing input variance but relies upon the system model and so potentially reduces the robustness of the closed-loop system. As a result, the authors are also investigating robust control and uncertainty in this context [30], [31].

\section{ACKNOWLEDGMENTS}

Supported by the UK Engineering and Physical Sciences Research Council (EPSRC) grant EP/M015637/1.

\section{REFERENCES}

[1] G. A. Dumont, "Closed-loop control of anesthesia-a review," IFAC Proceedings Volumes, vol. 45, no. 18, pp. 373-378, 2012.

[2] S. Bibian, C. R. Ries, M. Huzmezan, and G. Dumont, "Introduction to automated drug delivery in clinical anesthesia," European Journal of Control, vol. 11, no. 6, pp. 535-557, 2005.

[3] A. Gentilini, C. W. Frei, A. H. Glattfedler, M. Morari, T. J. Sieber, R. Wymann, T. W. Schnider, and A. M. Zbinden, "Multitasked closed-loop control in anesthesia," IEEE Engineering in Medicine and Biology Magazine, vol. 20, no. 1, pp. 39-53, 2001.

[4] G. A. Dumont, A. Martinez, and J. M. Ansermino, "Robust control of depth of anesthesia," International Journal of Adaptive Control and Signal Processing, vol. 23, no. 5, pp. 435-454, 2009. 
[5] P. C. Young, M. A. Behzadi, C. L. Wang, and A. Chotai, "Direct digital and adaptive control by input-output, state variable feedback pole assignment," International J. Control, vol. 46, pp. 1867-1881, 1987.

[6] C. J. Taylor, A. Chotai, and P. C. Young, "State space control system design based on non-minimal state-variable feedback: further generalisation and unification results," International Journal of Control, vol. 73, no. 14, pp. 1329-1345, 2000.

[7] C. J. Taylor, P. C. Young, and A. Chotai, True Digital Control: Statistical Modelling and Non-Minimal State Space Design. John Wiley and Sons, 2013.

[8] P. C. Young, M. Lees, A. Chotai, W. Tych, and Z. S. Chalabi, "Modelling and PIP control of a glasshouse micro-climate," Control Engineering Practice, no. 4, pp. 591-604, 1994.

[9] S. Quanten, P. McKenna, A. Van Brecht, A. Van Hirtum, P. C. Young, K. Janssens, and D. Berckmans, "Model-based PIP control of the spatial temperature distribution in cars," International Journal of Control, vol. 76, no. 16, pp. 16281634, 2003.

[10] C. J. Taylor, P. A. Leigh, A. Chotai, P. C. Young, E. Vranken, and D. Berckmans, "Cost effective combined axial fan and throttling valve control of ventilation rate," IEE Proceedings: Control Theory and Applications, vol. 151, no. 5, pp. 577584, 2004.

[11] E. M. Shaban, S. Ako, C. J. Taylor, and D. W. Seward, "Development of an automated verticality alignment system for a vibro-lance," Automation in Construction, vol. 17, no. 5, pp. 645-655, 2008.

[12] Q. Clairon, E. D. Wilson, R. Henderson, and C. J. Taylor, "Adaptive biomedical treatment and robust control," in 20th IFAC Triennial World Congress, Toulouse, France, July 2017.

[13] T. Hesketh, "State-space pole-placing self-tuning regulator using input-output values," IEE Proceedings: Control Theory and Applications, vol. 129, pp. 123-128, 1982.

[14] D. Simon, Optimal state estimation: Kalman, H-infinity, and nonlinear approaches. John Wiley \& Sons, 2006.

[15] R. C. Dorf and R. H. Bishop, Modern Control Systems. Pearson, 2011.

[16] H. W. Sorenson, Kalman filtering: theory and application. IEEE Press, 1985.

[17] S. J. Julier and J. K. Uhlmann, "A new extension of the Kalman filter to nonlinear systems," in International Symposium Aerospace/Defense Sensing, Simulation and Controls, vol. 3, no. 26, 1997, pp. 182-193.

[18] E. A. Wan and R. Van Der Merwe, "The unscented Kalman filter for nonlinear estimation," in IEEE Adaptive Systems for Signal Processing, Communications, and Control Symposium, 2000, pp. 153-158.

[19] N. J. Gordon, D. J. Salmond, and A. F. M. Smith, "Novel approach to nonlinear/non-Gaussian Bayesian state estimation," in IEE Proceedings: Radar and Signal Processing, vol. 140, 1993, pp. 107-113.
[20] M. S. Arulampalam, S. Maskell, N. Gordon, and T. Clapp, "A tutorial on particle filters for online nonlinear/non-Gaussian Bayesian tracking," IEEE Transactions on Signal Processing, vol. 50, pp. 174-188, 2002.

[21] J.-J. E. Slotine, J. K. Hedrick, and E. Misawa, "On sliding observers for nonlinear systems," Journal of Dynamic Systems, Measurement, and Control, vol. 109, pp. 245-252, 1987.

[22] V. I. Utkin, Sliding modes in control and optimization. Springer Science \& Business Media, 2013.

[23] S. S. Haykin, Kalman filtering and neural networks. Wiley Online Library, 2001.

[24] W. M. Wonham, "On the separation theorem of stochastic control," SIAM Journal on Control, vol. 6, pp. 312-326, 1968.

[25] E. D. Wilson, Q. Clairon, and C. J. Taylor, "Non-minimal state-space polynomial form of the Kalman filter for a general noise model," Electronics Letters, vol. 54, pp. 204-206, 2018.

[26] P. C. Young, "Self-adaptive Kalman filter," Electronics Letters, vol. 15, pp. 358-360, 1979.

[27] T. W. Schneider, C. Minto, P. L. Gambus, C. Andresen, D. B. Goodale, S. Shafer, and E. Youngs, "The influence of method of administration and covariates on the pharmacokinetics of propofol in adult volunteers," Anesthesiology, vol. 88, pp. 1170-82, 1998.

[28] J. Schüttler and H. Ihmsen, "Population pharmacokinetics of propofol. a multicenter study," Anesthesiology, vol. 92, no. 3, pp. 727-738, 2000.

[29] P. C. Young, Recursive Estimation and Time Series Analysis: An Introduction for the Student and Practitioner. Springer, 2011.

[30] E. D. Wilson, Q. Clairon, R. Henderson, and C. J. Taylor, "Robustness evaluation and robust design for proportionalintegral-plus control," International Journal of Control, pp. 113, 2018 (https://doi.org/10.1080/00207179.2018.1467042).

[31] E. D. Wilson, Q. Clarion, R. Henderson, and C. J. Taylor, "Dealing with observational data in control," Annual Reviews in Control, 2018 (https://doi.org/10.1016/j.arcontrol.2018.05.001). 